\title{
Malnutrizione associata a patologie, una problematica da risolvere
}

Mario Eandi ${ }^{\circ}$, Lorenzo Pradelli $^{\circ}$, Augusta Palmo*

REVIEW

\begin{abstract}
Disease-related malnutrition, the most frequent cause of undernourishment in industrialized countries, appears to be highly prevalent among western patient populations, interesting up to $60 \%$ of the hospitalized and almost one fifth of the persons attending to the general medicine service. There are several reasons for this high prevalence, among which pathology-related causes, inadequate presentation of the meals in hospitals and institutions, and low recognition of nutritional problems by health professionals. Several studies have shown that undernourished patients have higher morbidity and mortality rates than the general population and suffer of more and more severe complications, leading to prolonged hospital stays and worse clinical outcomes. This determines a much higher Health costs, estimated in an English study as a yearly additional burden of over 7 billion $£$ for the National Heath Service. Nutritional interventions, such as the administration of oral supplements or enteral tube feeding, are capable to increase body mass index (BMI), that is considered the most reliable indicator of the nutritional status, muscle strength and overall psycho-physical performance, determining an improved quality of life and a reduced need for medical assistance. These functional improvements go alongside with better clinical outcomes, as shown by a reduced mortality rate, shorter hospital stays and lower incidence of complications observed after supplementation. Studies that have analyzed the economical impact of nutritional supplementation in terms of cost-benefit have shown that the cost of enteral nutritional interventions is almost negligible when compared to the associated savings in the production of the health service, mainly determined by lower hospitalization rates, shorter hospital stays, reduced costs for the treatment of complications and for the consultation of specialists.

Greater awareness of the health professionals and more encouraging health policies toward nutritional interventions are required to reduce the clinical and economical impact of this unnecessarily wide-spread condition.
\end{abstract}

Farmeconomia e percorsi terapeutici 2002; 3 (3): 147 - 160

\section{INTRODUZIONE}

L'elevata prevalenza della malnutrizione nelle popolazioni di pazienti, sia di quelli ricoverati in ospedale sia curati a domicilio o in strutture residenziali, è un problema noto da tempo. Ciononostante, malgrado gli interventi di tipo nutrizionale atti a contrastare tale condizione siano ampiamente disponibili e di attuazione relativamente facile, stime recenti indicano che l'iponutrizione nei pazienti dei paesi occidentali rimane tuttora molto frequente, fortemente sottodiagnosticata e raramente trattata. La maggior parte delle persone accetta questo aspetto della malattia come se fosse "normale", qualcosa che non si può modificare. Ciò è in parte dovuto al fatto che sono stati compiuti pochi sforzi per creare consapevolezza su larga scala attorno all'argomento (1). Recentemente sono state invece pubblicate alcune rassegne della letteratura, in particolare dal gruppo della Dott.ssa Stratton dell'Università di Southampton (2-5), che hanno rialimentato il dibattito e che ci hanno fornito lo stimolo per un riesame dell'evidenza scientifica internazionale in materia. Presentiamo alcune indicazioni generali circa la prevalenza, le cause e le conseguenze della malnutrizione associata alle patologie, oltre ad una descrizione dei vantaggi clinici e funzionali derivanti da un supporto nutrizionale. Viene altresì trattata la questione dell'efficacia dei supporti nutrizionali enterali in termini di costo nella lotta alla malnutrizione associata a patologie.

\author{
${ }^{\circ}$ Farmacologia \\ Clinica, Università di \\ Torino \\ * Direttore della SC di \\ Dietetica e Nutrizione \\ Clinica, Azienda \\ Ospedaliera San \\ Giovanni Battista, \\ Torino
}




\section{Definizione del problema}

Per quanto si tratti di una problematica frequente, non esiste una definizione universale per malnutrizione.

Sebbene con il termine malnutrizione si intendano sia le carenze nutrizionali (iponutrizione) che l'eccesso di nutrienti (sovranutrizione/obesità), questo articolo si concentra unicamente sull'iponutrizione.

La causa più comune di iponutrizione nei paesi sviluppati è rappresentata dalle malattie e da fattori associati al processo patologico, tanto da essere arrivata a configurare un quadro nosologico a sé stante, denominato malnutrizione associata a patologie o iponutrione proteico-energetica.

L'iponutrizione si verifica se l'apporto di energia e nutrienti è insufficiente a soddisfare il fabbisogno energetico e nutrizionale di un individuo. Gli effetti avversi della malnutrizione sul corpo umano (forma, dimensione e composizione del corpo) e sulla funzionalità (fisica, psichica, comportamentale) sono tendenzialmente reversibili a seguito di un efficace intervento nutrizionale, sebbene in caso di malnutrizione prolungata alcuni effetti possano essere irreversibili.

Sono disponibili diversi criteri di valutazione del rischio di malnutrizione. Ad oggi non è stato individuato alcun criterio che possa essere definito ottimale per questo scopo e che sia stato universalmente accettato (6). Di conseguenza, sono stati sviluppati diversi indici e la percentuale di pazienti identificati come pazienti a rischio di malnutrizione nei diversi gruppi diagnostici varia a seconda dei criteri utilizzati per definire o diagnosticare questo stato. Tuttavia, gli studi presi in considerazione, effettuati in Europa e in altri paesi occidentali (ad esempio USA ed Australia), hanno rivelato che qualsiasi sia il criterio utilizzato la malnutrizione associata a patologie sembra essere un problema significativo in tutti i gruppi diagnostici, sia nel contesto ospedaliero, sia in comunità $(2,3)$.

L'indice utilizzato più frequentemente nei diversi studi nutrizionali condotti su adulti è il body mass index (BMI o indice di massa corporea); un valore di BMI inferiore a $20 \mathrm{~kg} / \mathrm{m}^{2}$ viene normalmente utilizzato per identificare lo stato di iponutrizione. Uno degli svantaggi di questo criterio, usato da solo, è la possibilità di sottostimare la gravità del problema. Vi possono essere molti individui a rischio di malnutrizione (con conseguente deterioramento delle funzionalità corporee) a causa di un calo ponderale sostanziale ma involontario (ad esempio $>10 \%$ del peso basale in 3-6 mesi), $\mathrm{i}$ quali possono mantenere un BMI ben al di sopra della soglia critica. D'altro canto, si os- servano alcuni individui sani con un BMI inferiore a $20 \mathrm{~kg} / \mathrm{m}^{2}$ (soprattutto tra i giovani adulti) che mantengono inalterate le funzionalità corporee.

Nei bambini è ancora più importante individuare precocemente il rischio di malnutrizione, in quanto i bambini possiedono riserve fisiologiche più limitate rispetto agli adulti, e la malnutrizione non determina solo un calo ponderale ed un problema di funzionalità, ma può causare rallentamento o arresto della crescita e dello sviluppo.

Sebbene la malnutrizione associata a patologie sia un problema che colpisce una percentuale considerevole di pazienti, tale problematica non viene individuata e non viene adeguatamente trattata con interventi di carattere nutrizionale nella maggior parte delle diagnosi.

\section{CAUSE DELLA MALNUTRIZIONE ASSOCIATA A PATOLOGIE}

La causa principale della malnutrizione, nei paesi occidentali, è costituita dalle malattie e dalle complicanze ad esse associate (7). In modo particolare, la malnutrizione è associata a: tumori maligni, malattie del tratto gastrointestinale, patologie renali, epatiche, cardiovascolari e neurologiche, alle infezioni ed ai disturbi provocati da traumi (tra cui le fratture e gli interventi chirurgici).

Sono molte le ragioni per cui i soggetti affetti da queste patologie o traumi sono ad alto rischio di malnutrizione, come riportato di seguito.

\section{Fattori associati alla patologia ed al trattamento farmacologico}

Scarso appetito a seguito della malattia, lesione o infezione

L'appetito può essere ulteriormente ridotto dalla terapia farmacologica e da alcuni degli effetti collaterali del trattamento (nausea, vomito, diarrea, mucosite, alterazioni del gusto)

L'assunzione di cibo può essere difficoltosa: il paziente non riesce a portare il cibo alla bocca, a masticare o a deglutire

- Il paziente è ansioso a causa della malattia, degli esami diagnostici e del trattamento; il contesto ospedaliero, insolito o spiacevole, può causare un'assunzione di cibo ridotta

- Affaticamento, affanno e minore tono muscolare possono limitare la capacità di preparare e consumare cibo. 
Inadeguata presentazione degli alimenti ai pazienti negli ospedali e negli Istituti di ricovero

- Scarsa palatabilità e presentazione inadeguata degli alimenti negli ospedali

- Inflessibilità dei servizi di ristorazione, che non forniscono alimenti familiari a determinate minoranze etniche

- Mancanza di assistenza nell'acquisto, nella preparazione e nell' aiuto al consumo dei pasti a domicilio o negli Istituti di ricovero

- Gli orari dei pasti si sovrappongono con esami clinici, visite ambulatoriali, trasferimenti dal medico o in ospedale

- Fornitura inadeguata di integrazione nutrizionale (supplementazione nutrizionale orale, nutrizione enterale per sonda, nutrizione parenterale) nei pazienti ad alto rischio di malnutrizione.

Mancanza di consapevolezza e non riconoscimento dei problemi dovuti alla malnutrizione negli ospedali e negli Istituti di ricovero

- Medici e infermieri carenti di conoscenze specifiche (o di formazione adeguata) circa i rischi associati alla malnutrizione ed i benefici della supplementazione nutrizionale

- Mancato riconoscimento della nutrizione come elemento fondamentale nel trattamento generale del paziente

- Mancato rilevamento dei pazienti a rischio di malnutrizione

- Insufficiente organizzazione dei servizi nutrizionali (ad esempio mancanza di un team che si occupi di tematiche nutrizionali, mancanza di politiche gestionali e di protocolli di trattamento inerenti la malnutrizione, scarso coordinamento tra ospedale e servizi sociali, mancanza di follow-up a domicilio).

Oltre a quanto già citato, i problemi psicologici quali povertà, solitudine, depressione $\mathrm{e}$ alcolismo possono contribuire all'insorgere della malnutrizione.

\section{INCIDENZAEPREVALENZADELLAMAL- NUTRIZIONEASSOCIATAAPATOLOGIE}

Utilizzando il criterio del BMI inferiore a 20 $\mathrm{kg} / \mathrm{m}^{2}$, la malnutrizione può essere percepita come un problema di sanità pubblica in Europa $(8,9)$. L'incidenza totale della malnutrizione nelle popolazioni europee corrisponde mediamente al 5\% per quanto riguarda gli uomini e al $15 \%$ per le donne, come illustrato nella Figura 1. Queste cifre possono essere considerate "elevate", ma sono basse se confrontate con la prevalenza della malnutrizione associata a specifiche patologie.

Il dato relativo alla malnutrizione associata a patologie varia a seconda della localizzazione o del contesto in cui si trovano i pazienti, ed in funzione del tipo di malattia, disturbo o condizione. Il numero di pazienti affetti da malnutrizione associata a patologia è più ampio tra $\mathrm{i}$ pazienti non ospedalizzati in carico ai medici di base che non tra quelli ricoverati in ospedale, semplicemente perché è qui che risiede la maggior parte della popolazione. Tuttavia la prevalenza a livello domiciliare è minore (fino al $40 \%$ negli anziani, fino al $13 \%$ nei pazienti ge-

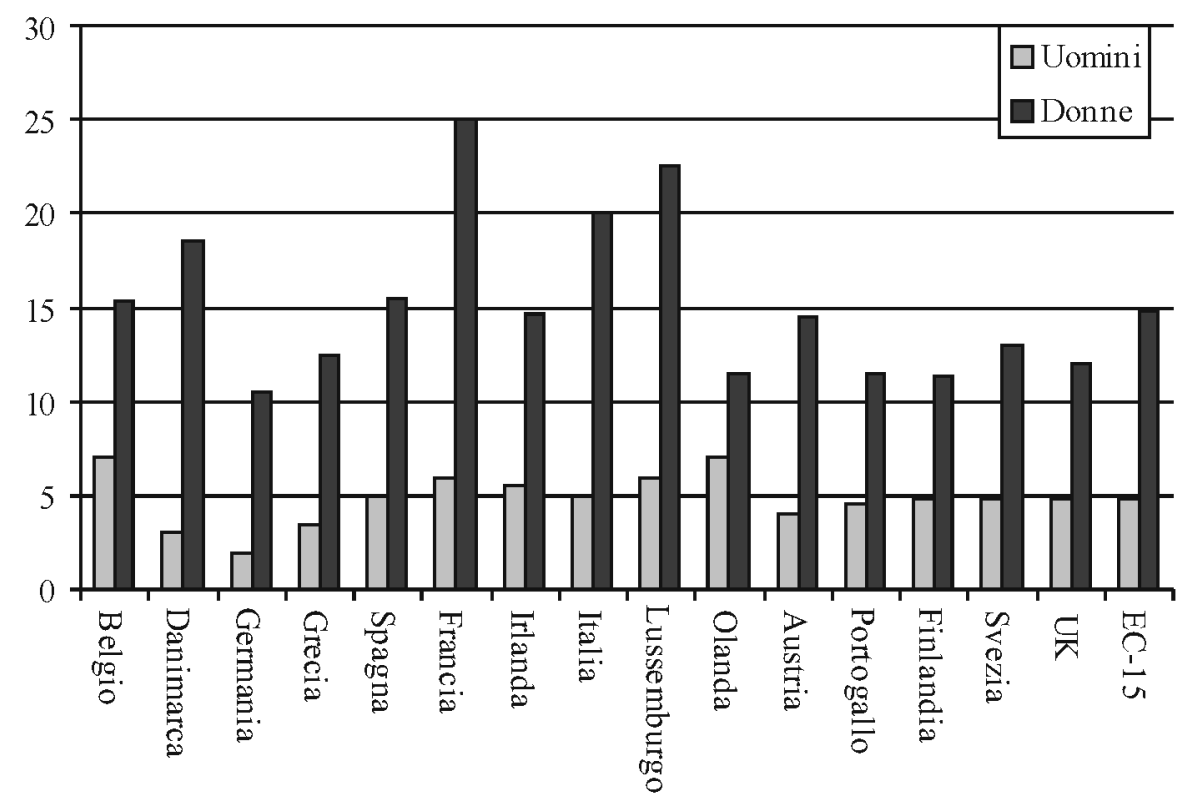

Figura 1.

Percentuale di popolazione nei paesi europei con BMI inferiore a 20 $\mathrm{kg} / \mathrm{m}^{2}$ (EC-15 rappresenta la media dei 15 paesi europei) 
nerici per i quali viene utilizzato l'indicatore BMI inferiore a $20 \mathrm{~kg} / \mathrm{m}^{2}$ ) rispetto alla situazione ospedaliera (2).

La prevalenza della malnutrizione è considerevolmente più alta nelle strutture sanitarie, tra cui gli Istituti di ricovero (fino all' $85 \%$ ) e gli ospedali (oltre il 60\%). Particolarmente allarmante è il peggioramento dello stato nutrizionale che si verifica negli ospedali, sia nei pazienti che si presentano ben nutriti che in quelli già scarsamente nutriti al momento del ricovero (3).

La malnutrizione associata alla patologia costituisce un problema rilevante in pazienti che presentano alcune specifiche condizioni cliniche. Infatti, gli studi condotti su gruppi di pazienti utilizzando il criterio del BMI inferiore a $20 \mathrm{~kg} / \mathrm{m}^{2}$ (o peso corporeo $<90 \%$ del peso ideale), come indicato nella Figura 2, hanno evidenziato la dimensione del problema della malnutrizione associata alla patologia con prevalenze che raggiungono le seguenti percentuali:

fino al $38 \%$ nei pazienti di medicina generale fino al $21 \%$ nei pazienti chirurgici

fino al $61 \%$ nei pazienti anziani in diverse condizioni cliniche

fino al $38 \%$ nei pazienti pediatrici

- fino al $50 \%$ nei pazienti con frattura alla testa del femore

fino al $36 \%$ nei pazienti oncologici fino al $31 \%$ nei pazienti con ictus cerebrovascolare (CVA/stroke)

fino al $60 \%$ nei pazienti con altri disturbi neurologici.

Gli altri gruppi di pazienti in cui, con altri indici di rilevazione, la malnutrizione è stata identificata come un problema significativo comprendono i pazienti con patologia gastrointestinale, epatica, insufficienza renale e quelli traumatizzati.

Molti di questi studi hanno escluso alcuni pazienti, ad esempio quelli troppo ammalati, che presentavano un deterioramento della sfera cognitiva o avevano perso conoscenza, o quelli affetti da altre malattie o ancora quelli che assumevano determinati farmaci. Di conseguenza, la prevalenza effettiva della malnutrizione in questi gruppi di pazienti rimane sconosciuta. È ragionevole ritenere che siano proprio i pazienti esclusi da questi studi quelli maggiormente a rischio di malnutrizione e che pertanto la reale prevalenza sia ancora superiore.

\section{CONSEGUENZEDELLAMALNUTRIZIONE ASSOCIATAAPATOLOGIE}

I dati raccolti mostrano in modo evidente che la malnutrizione associata a patologie determina una maggiore morbilità e una ridotta
Figura 2

Percentuali di pazienti adulti nei diversi gruppi diagnostici con un $B M I<20 \mathrm{~kg} / \mathrm{m}^{2}$

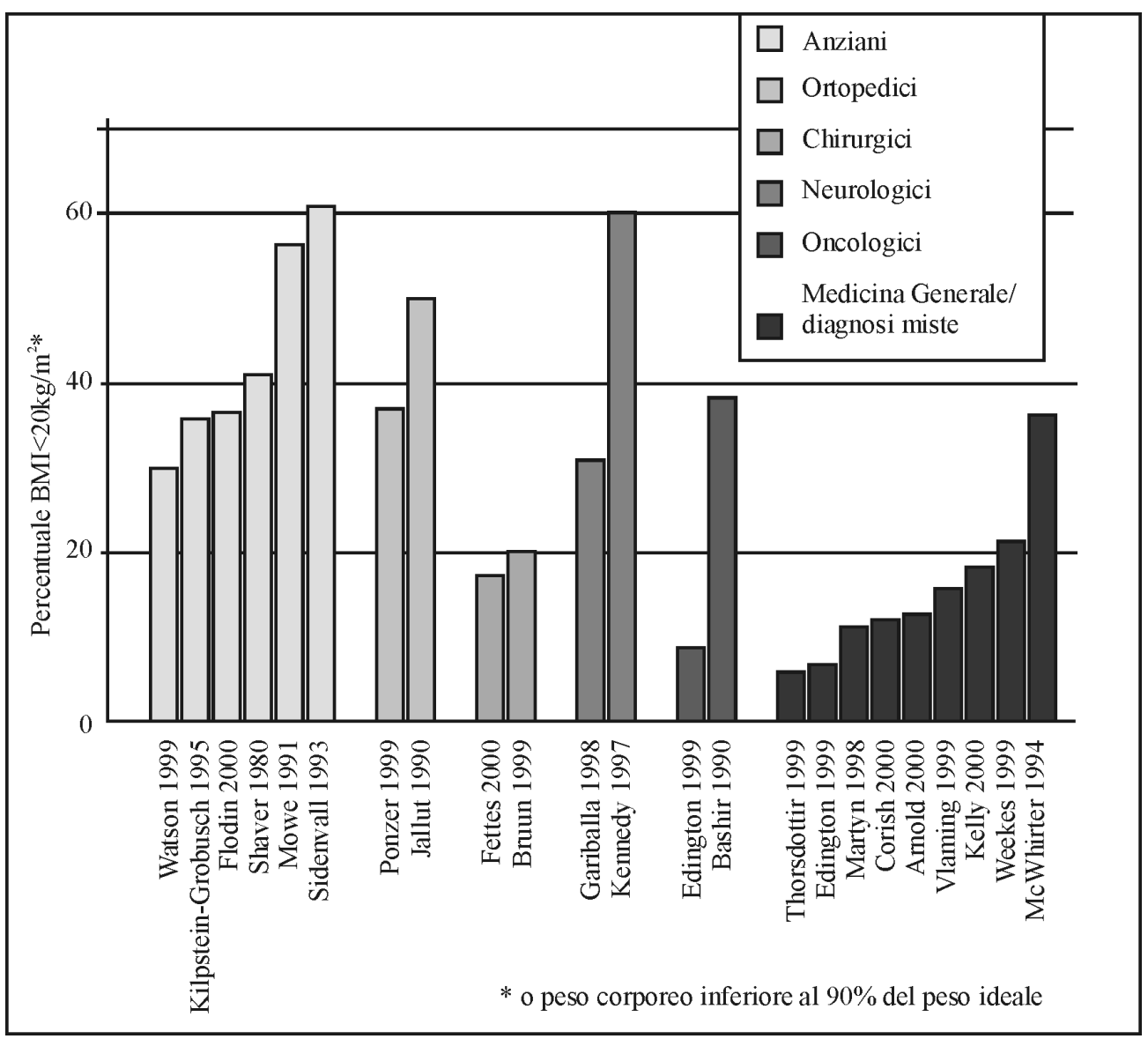


qualità di vita, oltre al deterioramento del tono muscolare e di altre funzioni corporee. Inoltre, la malnutrizione ha un impatto negativo sui risultati clinici, determinando l'aumento delle complicanze e del tasso di mortalità $(7,10-1)$. Le conseguenze cliniche e l'impatto economico associati alla malnutrizione sono riassunti nella Tabella 1.

L'impatto negativo della malnutrizione associata a patologie sulla risposta del paziente e sul ricovero ospedaliero determina il prolungamento della degenza e quindi un maggiore utilizzo delle strutture sanitarie, con un aumento anche importante dei costi. Le conseguenze economiche sono di ampia portata, in quanto l'utilizzo delle strutture sanitarie e i costi relativi aumentano considerevolmente nei pazienti malnutriti, sia in ospedale, che a livello domiciliare, nonché negli Istituti di ricovero (10-17).
Un' ampia indagine epidemiologica condotta nel Regno Unito in pazienti non ospedalizzati (2) ha riscontrato che i soggetti in stato di iponutrizione $\left(\mathrm{BMI}<20 \mathrm{~kg} / \mathrm{m}^{2}\right)$ presentavano maggiori percentuali di visite dal medico generico, di prescrizioni, di ricoveri ospedalieri e di morte, rispetto ai soggetti con un BMI compreso tra 20 e $25 \mathrm{~kg} / \mathrm{m}^{2}$.

In maniera analoga, $\mathrm{i}$ dati provenienti dagli Stati Uniti indicano che i soggetti con un BMI diverso dal range considerato "ideale" devono sostenere maggiori spese sanitarie, sia che si tratti di soggetti in stato di "iponutrizione", di "ipernutrizione" o di “obesità". Un'analisi effettuata nel 1994 (19) ha evidenziato che la spesa sanitaria annuale aumentava progressivamente con la diminuzione del BMI, variando da US\$ 1.850 per una donna con un BMI pari a $21 \mathrm{~kg} / \mathrm{m}^{2}$ fino a US\$2.350 per una donna con un BMI di $15 \mathrm{~kg} / \mathrm{m}^{2}$. L'aumento della spesa

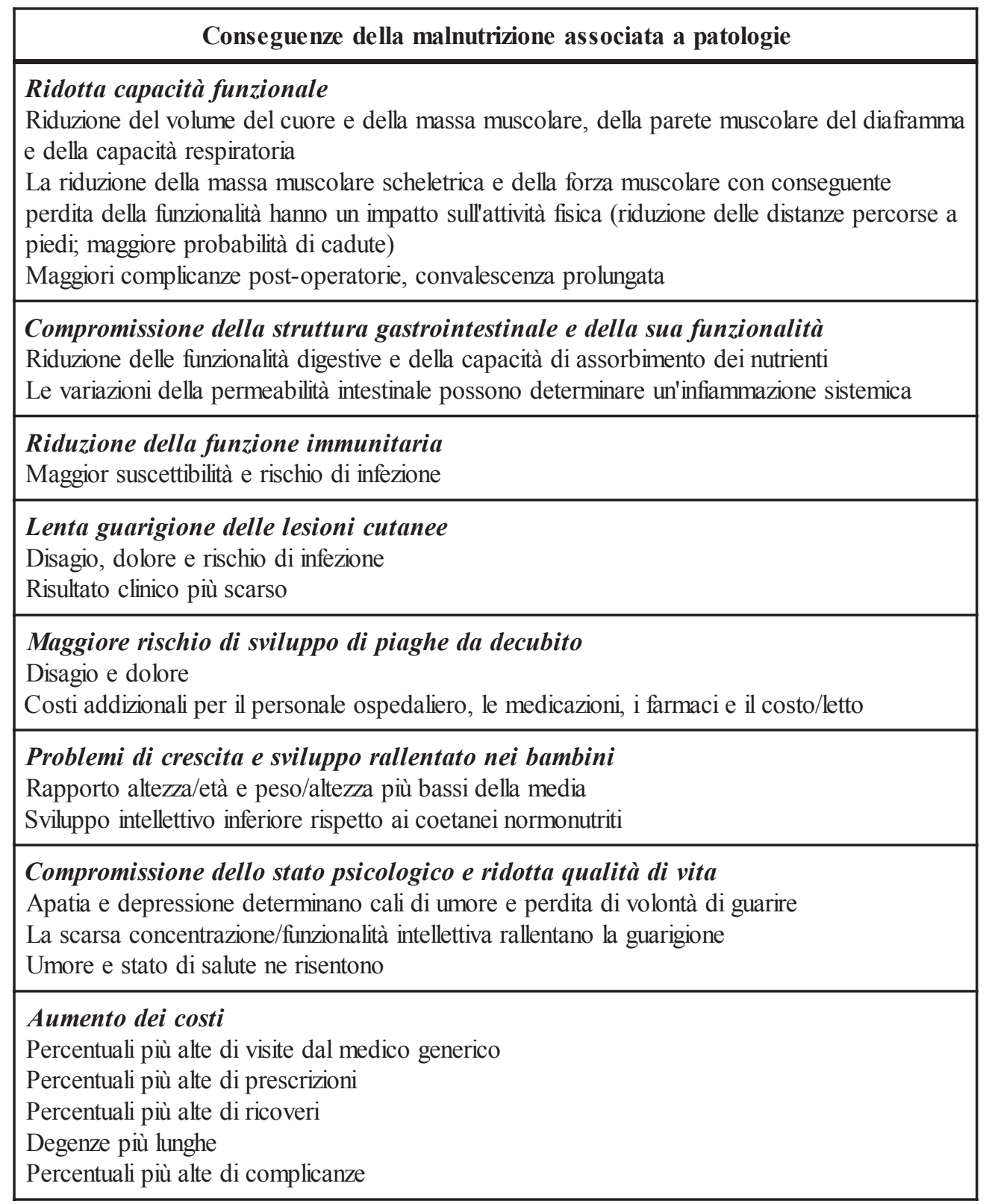

Tabella 1

Conseguenze della malnutrizione associata a patologie 
sanitaria annuale era più marcato negli uomini (da US\$ 1.300 per un soggetto con BMI di 21 $\mathrm{kg} / \mathrm{m}^{2}$ a circa US\$ 3.250 per un soggetto con BMI di $15 \mathrm{~kg} / \mathrm{m}^{2}$ ). Un aumento della spesa sanitaria è stato registrato anche a carico dei soggetti in sovrappeso o obesi (US\$ 1.700 per un soggetto con BMI di $39 \mathrm{~kg} / \mathrm{m}^{2}$ ).

Le conseguenze economiche della malnutrizione associata a patologie nei pazienti ospedalizzati sono state stimate anche in un altro studio condotto negli USA. In quest'indagine (20) è stato evidenziato come i costi ospedalieri per i pazienti malnutriti fossero dal 35 al $75 \%$ più elevati dei costi sostenuti per i pazienti normonutriti. Allo stesso modo, in uno studio inglese condotto su pazienti seguiti dai reparti di medicina (14) è stato calcolato che i soggetti a rischio di malnutrizione determinavano costi ospedalieri significativamente superiori (circa 1.000 sterline per paziente), con degenze ospedaliere significativamente prolungate. Successivamente, in un' analisi più complicata condotta dal gruppo Nutrition Screening Initiative (21), è stato calcolato che sarebbe stato possibile risparmiare ingenti somme di denaro se fossero stati effettuati interventi nutrizionali enterali mirati a prevenire le conseguenze della malnutrizione, riducendo quindi il livello ed il tipo di complicanze nonché le degenze ospedaliere.

In Inghilterra, è stato stimato che il costo aggiuntivo del trattamento di pazienti con un $\mathrm{BMI}<20 \mathrm{~kg} / \mathrm{m}^{2}$ potrebbe ammontare a circa 7,3 milioni di sterline/anno per 100.000 pazienti. Inoltre, dalle prime stime è risultato che sarebbe stato possibile ottenere un risparmio per il Servizio Sanitario Nazionale inglese di $266 \mathrm{mi}-$ lioni di sterline/anno, se fosse stato somministrato un adeguato supporto nutrizionale ai pazienti malnutriti (costi 1992).

\section{EFFICACIA CLINICA DEL SUPPORTO NUTRIZIONALENELLAMALNUTRIZIONE ASSOCIATAAPATOLOGIE}

Numerosi studi individuali hanno mostrato risultati significativi del supporto nutrizionale in molte patologie. Tuttavia solo ora è stato effettuato un riesame di tutti i lavori clinici condotti in merito, con l'obiettivo di fornire chiare indicazioni sulla rilevanza generale del supporto nutrizionale.

I supplementi nutrizionali orali (SNO) vengono utilizzati per migliorare l'intake nutrizionale nei pazienti che non sono in grado di soddisfare le loro richieste energetiche e nutrizionali con la sola assunzione degli alimenti, nonostante uno specialista abbia consigliato loro di aumentare l'apporto energetico e proteico. I SNO vengono utilizzati negli ospe- dali europei in molti pazienti affetti da un'ampia gamma di patologie. Molti pazienti che assumono gli SNO in ospedale continuano ad effettuare l'integrazione una volta tornati a casa. Due revisioni $(2,7)$ sistematiche della letteratura internazionale (che comprendono un totale di 136 trial e di 6.279 pazienti) hanno confermato l'efficacia ed i vantaggi dell'impiego degli SNO nei pazienti ospedalizzati e non, affetti da un'ampia gamma di patologie ed in diverse condizioni cliniche. Gli studi oggetto delle review di lavori clinici internazionali comprendevano pazienti affetti dalle seguenti patologie: BPCO, patologie epatiche, HIV/ AIDS, tumori, patologie chirurgiche, fibrosi cistica, morbo di Crohn, patologia neurologica, ustioni, traumi ortopedici, ortognatici, patologia gastrointestinale, e altre.

L'utilizzo dei SNO negli ospedali ha determinato un miglioramento del rapporto tra energia totale e assunzione di nutrienti nel $97 \%$ dei trial (con significatività statistica nel $61 \%$ degli studi clinici randomizzati e nel $93 \%$ degli studi clinici non randomizzati), mentre non ha ridotto l'assunzione di cibo in maniera evidente. In alcuni studi su pazienti ospedalizzati, al contrario, l'impiego degli SNO ha determinato l'aumento dell' assunzione di cibo. In situazioni extra-ospedaliere (a domicilio o in Istituto di ricovero), 27 studi randomizzati e controllati hanno mostrato un incremento di tale valore durante la fase di integrazione (significativo in 18 studi). L'aumento generale in termini di assunzione era equivalente al $67 \%$ dell'energia del supplemento nutrizionale consumato, pur con ampie variazioni, a seconda dello stato della malattia e del BMI, essendo più marcata nei pazienti con un BMI $<20 \mathrm{~kg} / \mathrm{m}^{2}$.

I risultati di questi studi evidenziano che l'aumento dell' intake dovuto agli SNO favorisce il ripristino del peso corporeo ideale e aumenta la massa muscolare.

La supplementazione orale ha determinato miglioramenti dello stato nutrizionale nell' $83 \%$ dei trial condotti in ospedale (il 62\% dei trial ha evidenziato miglioramenti statisticamente significativi), in cui i pazienti avevano acquistato più peso o perso meno peso rispetto al gruppo di controllo. Inoltre, i miglioramenti in termini di peso corporeo e di valori antropometrici hanno avuto una frequenza simile nei trial che comprendevano pazienti con BMI medio inferiore a $20 \mathrm{~kg} / \mathrm{m}^{2}$ ed in quelli con BMI superiore a $20 \mathrm{~kg} / \mathrm{m}^{2}$.

Negli Istituti di ricovero ed a domicilio, l'incremento ponderale variava considerevolmente a seconda della durata e dell'entità della supplementazione, oltre che dello stato della malattia. Aumenti significativi a livello di massa muscolare e massa grassa sono stati segnalati nel 50\% degli studi clinici condotti a domi- 
cilio in cui era disponibile la misurazione della composizione corporea. I miglioramenti dello stato nutrizionale erano più marcati nei pazienti con un BMI inferiore a $20 \mathrm{~kg} / \mathrm{m}^{2}$.

Inoltre, gli SNO migliorano la crescita nei bambini con ritardo dello sviluppo. Sette degli undici studi condotti su pazienti negli Istituti di ricovero ed a domicilio hanno mostrato miglioramenti significativi della crescita nei bambini con ritardo dello sviluppo ed affetti da morbo di Crohn o fibrosi cistica.

Come prevedibile dal miglioramento degli intake e dei parametri antropometrici, l'impiego degli SNO nei pazienti a rischio di malnutrizione associata a patologie può determinare una moltitudine di vantaggi funzionali, che variano a seconda della malattia e/o delle condizioni generali del paziente.

In ospedale, gli SNO hanno determinato miglioramenti della funzionalità corporea generale nel 79\% degli studi (significativi nel 53\% dei casi), specificamente quando si riscontravano aumenti a livello di assunzione di energia e di peso corporeo. I vantaggi individuati comprendono l'aumento del tono muscolare, la capacità di percorrere maggiori distanze a piedi, il miglioramento della salute mentale e fisica e dei livelli di attività, con un impatto complessivo molto positivo sulla qualità di vita.

Gli studi clinici condotti su pazienti a domicilio hanno evidenziato che i vantaggi funzionali si riscontravano più frequentemente nei pazienti con un BMI inferiore a $20 \mathrm{~kg} / \mathrm{m}^{2}$ piuttosto che in quelli con BMI superiore a 20 $\mathrm{kg} / \mathrm{m}^{2}$. I vantaggi funzionali (osservati in 59 studi) variavano a seconda della malattia/condizione generale, come ad esempio:

- Aumento del tono muscolare, maggiori distanze percorse a piedi e miglior qualità di vita nei pazienti affetti da broncopneumopatia cronica ostruttiva (BPCO)

- Crescita migliorata nei bambini affetti da fibrosi cistica

- Minor numero di cadute e svolgimento di un maggior numero di attività quotidiane negli anziani

- Miglioramento generale della risposta immunitaria nei pazienti HIV-positivi.

Come esito finale dei vari vantaggi esposti, l'impiego degli SNO può significativamente migliorare il risultato clinico in molti gruppi di pazienti.

La maggior parte $(86 \%)$ dei lavori clinici di valutazione degli outcome nei pazienti ospedalizzati ha dimostrato effetti positivi della supplementazione con gli SNO (risultati statisticamente significativi nel 50\% degli studi), evidenziando in particolare la riduzione delle percentuali di mortalità, della durata media della degenza ospedaliera e delle percentuali di complicanze, risultati più evidenti nei pazienti a rischio di malnutrizione.

Nei pazienti trattati a domicilio o in Istituti di ricovero, l'interruzione della supplementazione per un periodo variabile tra 8 giorni e 1 anno ha determinato la riduzione dell'intake energetico totale, causando calo ponderale (o minore incremento ponderale) e riduzione della funzionalità. In alcuni studi (in pazienti con BPCO ed in pazienti con fibrosi cistica) i miglioramenti sono rimasti costanti, al di sopra del valore basale, per 2-3 mesi dall'interruzione della supplementazione nutrizionale.

Generalmente, il ricorso allo specialista per migliorare ed aumentare l'intake energetico e proteico, è raccomandato come primo intervento di carattere nutrizionale, ancor prima di iniziare ad assumere i supplementi nutrizionali per os. Tuttavia, l'efficacia di questo tipo di intervento non è ancora ben chiara. Succede spesso che i pazienti semplicemente non assumano i normali pasti che dovrebbero, e tanto meno gli alimenti addizionali consigliati dallo specialista.

Inoltre, la ricerca suggerisce che gli SNO possono essere più efficaci rispetto alle raccomandazioni alimentari dello specialista. Infatti, una piccola rassegna su 4 studi clinici di confronto tra quanto consigliato dallo specialista e la supplementazione con gli SNO ha rivelato che dopo 3 mesi i pazienti che assumevano gli SNO presentavano un incremento ponderale significativamente superiore rispetto a quelli che avevano ricevuto le raccomandazioni alimentari dello specialista, probabilmente grazie al fatto che l'assunzione di energia era significativamente aumentata nel gruppo SNO.

I pazienti che non sono in grado di soddisfare il proprio fabbisogno nutrizionale per via orale, attraverso la normale dieta ed i supplementi nutrizionali per os, possono trovare beneficio da una nutrizione clinica enterale per sonda. La nutrizione enterale per sonda (NES) è indicata come unica fonte di nutrizione nei pazienti che non possono mangiare ma che mantengono integra la funzionalità del tratto gastrointestinale.

L'efficacia della NES nelle diverse diagnosi, nei diversi range di età e contesti sanitari, è stata evidenziata in una review (5) che ha preso in considerazione 108 studi clinici, per un totale di 2.720 pazienti. I dati suggeriscono che la NES rappresenta un metodo sicuro e pratico per raggiungere determinati apporti nutrizionali e che il suo utilizzo è in crescita nei paesi europei, in modo particolare quello a lungo termine, nei pazienti non ospedalizzati, che si trovano a domicilio o ricoverati in Istituti o Case di cura. 
La NES può efficacemente migliorare l'apporto totale di energia e di nutrienti nei pazienti di tutte le età, ricoverati in ospedale $o$ in Istituti di ricovero o a domicilio, affetti da diverse patologie. In 42 lavori clinici sono stati riscontrati miglioramenti in termini di apporto totale di energia e nutrienti mediante la NES (i dati ottenuti sono risultati statisticamente significativi nel $67 \%$ dei casi). In aggiunta all'utilizzo indispensabile della NES nei pazienti che non sono in grado di assumere cibo, questa modalità di trattamento si è dimostrata notevolmente efficace se utilizzata come supplemento alla normale alimentazione.

Sono stati ampiamente documentati miglioramenti in termini di peso corporeo, composizione e crescita corporea nei bambini con ritardo dello sviluppo. In ben 66 lavori clinici esaminati venivano evidenziati miglioramenti in termini di incremento ponderale dei pazienti trattati con nutrizione enterale per sonda, rispetto ai pazienti controllo; i dati erano significativi nel 55\% dei casi. Nel 75\% dei lavori clinici la nutrizione enterale per sonda si è dimostrata efficace nell'aumentare la massa magra e quella grassa. Inoltre, in 14 trial clinici la NES è stata associata a miglioramenti della crescita nei bambini con ritardo dello sviluppo.

L'utilizzo della NES può determinare numerosi vantaggi funzionali, a seconda della tipologia di paziente. Tra gli studi che valutavano la funzionalità dei pazienti ospedalizzati, il $90 \%$ ha segnalato vantaggi in termini di miglioramento della funzionalità immunitaria e respiratoria, di mobilità, di tono muscolare e di guarigione delle lesioni, con conseguente miglioramento della qualità di vita. Allo stesso modo, negli studi di valutazione della funzionalità condotti su pazienti non ospedalizzati, l' $81 \%$ ha riportato miglioramenti (Tabella 2). Di particolare rilevanza sono i miglioramenti dei risultati clinici associati alla

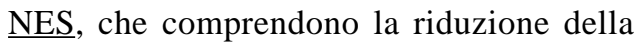
morbilità e della mortalità. In ospedale, il 79\% degli studi condotti per valutare la risposta clinica ha segnalato miglioramenti. La risposta clinica è più difficile da valutare nei pazienti non ospedalizzati, in quanto per molti di questi la NES può essere considerata come terapia di "mantenimento in vita" e l'inclusione di un gruppo di controllo non sarebbe eticamente sostenibile. Ciò nonostante, sono stati segnalati benefici in oltre il $70 \%$ degli studi condotti allo scopo di valutare l'outcome clinico, tra cui la riduzione delle percentuali di ricovero e di degenza ospedaliera, quando questa era necessaria, oltre a una riduzione della mortalità

(vedi Tabella 2).
EFFICACIA INTERMINIDI COSTO-BENEFICIO DELLA SUPPLEMENTAZIONE NUTRIZIONALE

I miglioramenti della funzionalità e degli outcome clinici possono incidere notevolmente sulla riduzione dei costi, grazie al minore consumo di risorse sanitarie, sia in ospedale che negli Istituti di ricovero. In momenti come questo, in cui il budget a disposizione si riduce sempre più, diventa ancor più importante dimostrare che l'utilizzo di terapie nutrizionali non solo risulta clinicamente efficace, ma anche economicamente vantaggioso. I risparmi possono derivare da diversi fattori, conseguenti all'intervento di tipo nutrizionale. E' intuitivo che la più rapida guarigione delle lesioni, la riduzione delle complicanze cliniche, la riduzione della degenza e del numero di riospedalizzazioni successive possono determinare risparmi sostanziali per il sistema sanitario; tuttavia, la reale efficacia in termini di costo-beneficio è difficile da quantificare, dati i problemi che si verificano nella valutazione dei costi stessi e nel trasportare i risultati ottenuti dagli studi clinici nella pratica routinaria.

Ad ogni modo, le valutazioni retrospettive indicano che i risparmi potenziali sono di portata troppo ampia per essere ignorati.

Una review americana (21) basata sull'evidenza dell'efficacia clinica ed economica della nutrizione enterale, intesa sia come nutrizione orale che per sonda, ha concluso che queste forme di supplementazione nutrizionale sono altamente efficaci in termini economici:

"La somministrazione regolare ed appropriata dei prodotti nutrizionali ( per sonda e per os) nel trattamento di un'ampia gamma di condizioni cliniche [...] migliora lo stato di salute determinando la riduzione dei costi a carico del Servizio Sanitario Nazionale."

"I risparmi sono determinati da diversi fattori, tra cui la riduzione delle complicanze cliniche sia in termini di gravità che di numerosità, la riduzione della degenza ospedaliera, la riduzione delle riospedalizzazioni, il miglioramento nella guarigione delle lesioni, il miglioramento della risposta immunitaria ed il maggiore utilizzo della via di somministrazione enterale rispetto a quella parenterale.

Questi risparmi compensano ampiamente il costo sostenuto per integrare l'alimentazione dei pazienti'.

In questa review è stato inoltre calcolato che:

"l'utilizzo coerente ed appropriato della terapia nutrizionale [...] avrebbe determinato un risparmio per il sistema sanitario nazionale superiore a 150 milioni di dollari nel 1994, determinando una previsione di risparmio pari a 1,3 miliardi di dollari tra il 1996 e il 2002." 


\begin{tabular}{|c|c|}
\hline Condizione & Vantaggi funzionali/clinici \\
\hline $\mathrm{BPCO}$ & $\begin{array}{l}\text { Miglioramenti: } \\
\text { funzionalità respiratoria (pressione inspiratoria ed espiratoria, minore } \\
\text { affanno) } \\
\text { maggiore tono muscolare } \\
\text { maggiore resistenza allo sforzo fisico } \\
\text { riduzione della frequenza e della durata delle degenze dovute a } \\
\text { complicanze polmonari }\end{array}$ \\
\hline Patologia epatica & Minore tasso di mortalità e minor gravità delle complicanze \\
\hline HIV/AIDS & Migliore qualità di vita, vantaggi a livello immunologico \\
\hline Cancro & $\begin{array}{l}\text { Miglioramenti dei parametri immunologici } \\
\text { Migliore risposta alla chemioterapia } \\
\text { Miglioramento della performance } \\
\text { Migliore qualità di vita o minore scadimento della qualità di vita } \\
\text { Minore tasso di mortalità }\end{array}$ \\
\hline Chirurgia & $\begin{array}{l}\text { Più rapida ripresa della funzione intestinale, attenuazione della } \\
\text { permeabilità gastrointestinale. } \\
\text { Guarigione più rapida delle ferite } \\
\text { Benefici a livello immunologico } \\
\text { Minore quantità di complicanze postoperatorie, dimissioni più rapide } \\
\text { Minore utilizzo di nutrizione parenterale }\end{array}$ \\
\hline Fibrosi cistica & $\begin{array}{l}\text { Miglioramento delle prestazioni funzionali nelle attività quotidiane e } \\
\text { miglioramento della qualità di vita } \\
\text { Miglioramento della funzionalità polmonare } \\
\text { Migliori risultati clinici } \\
\text { Riduzione degli episodi di polmoniti }\end{array}$ \\
\hline Ustioni & $\begin{array}{l}\text { Degenze puù brevi } \\
\text { Minore quantità di infezioni delle ferite e delle lesioni }\end{array}$ \\
\hline Ortopedia & $\begin{array}{l}\text { Minor durata della riabilitazione, degenze più brevi, minore tasso di } \\
\text { mortalità }\end{array}$ \\
\hline Medicina generale & $\begin{array}{l}\text { Miglioramento dei parametri immunologici } \\
\text { Maggiore assorbimento intestinale } \\
\text { Migliore funzionalità gastrointestinale } \\
\text { Miglioramento della qualità di vita } \\
\text { Minore tasso di mortalità }\end{array}$ \\
\hline Gastroenterologia & $\begin{array}{l}\text { Più rapida ripresa della funzionalità gastrointestinale } \\
\text { Minori riacutizzazioni in corso di morbo di Crohn } \\
\text { Riduzione delle dosi di steroidi somministrati ai bambini } \\
\text { Migliori livelli circolanti di vitamine } \\
\text { Riduzione delle complicanze } \\
\text { Minore tasso di mortalità }\end{array}$ \\
\hline $\begin{array}{l}\text { Malati critici } \\
\text { /traumatizzati }\end{array}$ & $\begin{array}{l}\text { Miglioramento dei parametri immunologici } \\
\text { Minori complicanze di natura infettiva } \\
\text { Minore morbilità settica } \\
\text { Attenuazione della permeabilità intestinale } \\
\text { Tendenza verso una migliore risposta neurologica } \\
\text { Minore tasso di mortalità }\end{array}$ \\
\hline Pediatria & $\begin{array}{l}\text { Miglioramento dello stato clinico } \\
\text { Minore utilizzo di nutrizione parenterale }\end{array}$ \\
\hline Anziani & $\begin{array}{l}\text { Miglioramento della risposta immunitaria } \\
\text { Miglioramento della mobilità } \\
\text { Diminuzione dello stato confusionale } \\
\text { Riduzione delle dimensioni delle piaghe da decubito } \\
\text { Riduzione della degenza ospedaliera }\end{array}$ \\
\hline Patologia renale & Riduzione dei giorni di degenza ospedaliera \\
\hline
\end{tabular}

Tabella 2

Effetti della NES sugli outcome clinici, suddivisi per aree patologiche 
Efficacia in termini di costo-beneficio dei supplementi nutrizionali per os in pazienti ospedalizzati

Molte analisi retrospettive effettuate sui costi hanno suggerito che con un appropriato impiego degli SNO è possibile ottenere dei risparmi significativi in termini di riduzione della degenza ospedaliera e della spesa prevista per il trattamento delle complicanze. È stato calcolato che sarebbe possibile operare un risparmio per il servizio sanitario inglese di oltre 266 milioni di sterline/anno se fosse impiegata un'appropriata supplementazione nutrizionale nei pazienti malnutriti (stima conservativa basata su un intervento di tipo nutrizionale che ridurrebbe la degenza ospedaliera di 5 giorni nel $10 \%$ dei pazienti ospedalizzati).

\section{Efficacia in termini di costo-beneficio dei supplementi nutrizionali per os nei pazienti non ospedalizzati}

I dati inerenti l'efficacia in termini di costobeneficio nei pazienti non ospedalizzati risultavano più limitati, ma comunque sufficienti a dimostrare che gli SNO determinano migliori outcome funzionali, una riduzione delle visite dal medico generico, delle prescrizioni, delle complicanze e del numero di ospedalizzazioni, con degenze più brevi e miglioramenti dello stato clinico.

È quindi probabile che, nei pazienti con malnutrizione associata a patologie, gli $\underline{\mathrm{SNO}}$ possano rivelarsi costo efficaci. Nei pazienti che necessitano di una forma qualsiasi di supplementazione nutrizionale a lungo termine, è risultato che il trattamento a domicilio è considerevolmente più costo efficace rispetto al trattamento ospedaliero.

\section{Efficacia in termini di costo-beneficio della nutrizione clinica enterale per sonda nei pa- zienti ospedalizzati}

Sono disponibili dati molto interessanti che suggeriscono molto chiaramente che la nutrizione enterale per sonda può determinare la riduzione dei costi per i pazienti con malnutrizione associata a patologie. Specificamente, utilizzando i dati raccolti in maniera prospettica, in Inghilterra è stato stimato che sarebbe possibile ottenere enormi risparmi se i pazienti con patologie neurologiche potessero assumere una forma di nutrizione enterale per sonda (NES) sin dai primi giorni dell'ospedalizzazione, grazie alla riduzione delle complicanze e della degenza ospedaliera che ne deriverebbe. In uno dei primi studi condotti in un gruppo di pazienti di medicina è stato stimato un risparmio potenziale superiore alle 4.519 sterline/paziente (costi relativi al 1993 in Inghilterra) se la NES fosse introdotta nei primi giorni di degenza, in modo da evitare lunghi periodi di digiuno o di iponutrizione.

In quattro studi sono state effettuate valutazioni prospettiche circa il costo della nutrizione enterale per sonda (NES). In due studi, condotti tra i pazienti pediatrici, i risparmi sono risultati evidenti. Nei bambini con patologie critiche, l'impiego della NES in sostituzione della nutrizione parenterale (NP) ha determinato un risparmio di 2.701 dollari /paziente. Allo stesso modo, l'introduzione di un protocollo per promuovere l'uso appropriato della NES nei bambini con diarrea cronica intrattabile ha determinato un risparmio di 14.750 dollari/paziente, dovuto alla riduzione della degenza ospedaliera e del ricorso alla NP.

Inoltre, negli adulti che hanno subito un intervento chirurgico, è stato ipotizzato che la nutrizione enterale possa ridurre la necessità di NP e le spese relative. Questa ipotesi è stata confermata in uno studio in cui venivano fornite delle stime economiche. L'impiego della NES nel periodo immediatamente successivo all'intervento chirurgico aveva ridotto i costi ospedalieri (di circa 1.000 sterline/paziente) in confronto al gruppo di controllo (circa 2.000 sterline/paziente) che assumeva placebo. L'utilizzo di formulazioni enterali specifiche per patologia può risultare efficace in termini di costo-beneficio, laddove queste ultime determinino un ulteriore miglioramento degli outcome clinici. Infatti, due metanalisi su studi condotti in pazienti critici e nel post-operatorio, che assumevano formulazioni enterali arricchite con nutrienti in grado di modulare i parametri immunologici ed infiammatori, hanno evidenziato l'efficacia della NES in termini di costo, in quanto aveva determinato miglioramenti della risposta clinica e una riduzione dell'utilizzo delle risorse interne (umane ed economiche), ovvero riduzione della degenza ospedaliera.

Efficacia in termini di costo-beneficio della nutrizione clinica enterale per sonda nei pazienti non ospedalizzati

Per pazienti trattati in Istituto di ricovero o a domicilio è stato stimato che mediante l'utilizzo della supplementazione nutrizionale è possibile evitare l'ospedalizzazione, con risparmi annuali consistenti (nel 1995 oltre 90 milioni di sterline/anno in Inghilterra per la NES e la NP). Infatti, se la prevalenza di pazienti che assumono la nutrizione enterale per sonda (NES) domiciliare in Inghilterra fosse di circa 
15.000 entità, e ogni paziente venisse trattato a domicilio anziché in ospedale per un anno, si potrebbe ottenere un risparmio superiore a 1 miliardo di sterline (presupponendo un costo ospedaliero di 200 sterline/giorno per 365 giorni senza costi dovuti alla nutrizione). Sebbene i pazienti che assumono la NES a domicilio vengano occasionalmente ricoverati, gli studi e le ricerche effettuate hanno individuato una percentuale minore dell' $1 \%$ di ricoveri. Perciò i risparmi e la liberazione di risorse di cui beneficerebbe il sistema sanitario sono verosimilmente consistenti.

\section{Confronto dell'efficacia in termini di costo- beneficio della nutrizione clinica enterale rispetto alla nutrizione parenterale}

La nutrizione parenterale (NP) è chiaramente un trattamento più costoso della nutrizione enterale (NES), sia a livello ospedaliero che domiciliare. I costi della NP sono costituiti da diversi elementi, tra cui le soluzioni di NP, le sonde, le pompe, le medicazioni, oltre che dagli esami di laboratorio e dal personale specialistico necessari per effettuare il monitoraggio e il trattamento delle complicanze. I costi della nutrizione enterale per sonda (NES) sono notevolmente inferiori, essendo più contenuti i costi dell'equipaggiamento necessario e del monitoraggio. Ad esempio, già nel 1985 era stato calcolato che il costo medio della NP per paziente ospedalizzato negli USA ammontava a circa 500 dollari, contro i 30 dollari della NES. È probabile che i risparmi associati al miglioramento della risposta clinica, osservati a seguito dell'utilizzo della NES (ovvero riduzione della percentuale di complicanze, riduzione della degenza in terapia intensiva ed in ospedale, tempi di dimissione più rapidi), superino di gran lunga i costi relativamente contenuti del prodotto stesso. Ciò è meno probabile che si verifichi con la NP a causa del costo maggiore di questa terapia e del trattamento delle complicanze associate alla nutrizione per via endovenosa.

In sintesi, "nei pazienti che mantengono la funzionalità gastrointestinale, il motto "Se l'intestino funziona, usalo" risponde perfettamente alle esigenze fisiologiche e naturali dell'organismo e permette inoltre un notevole risparmio economico".

\section{CONCLUSIONI}

La malnutrizione associata a patologie rappresenta un problema di salute pubblica globale. Valutando il problema in termini generali ci si rende conto che la malnutrizione associata a patologie è molto più comune di quanto si possa pensare e determina problemi ancor più gravi di quanto si creda. Ridotti tassi di sopravvivenza, minore capacità funzionale, degenze ospedaliere prolungate, maggior numero di prescrizioni per le concomitanti terapie farmacologiche sono solo alcuni dei problemi riscontrati.

La disponibilità di una tale mole di informazioni sul problema malnutrizione costituisce uno stimolo e un' opportunità per iniziare a risolvere il problema. Il riconoscimento e la comprensione di tale problematica da parte degli specialisti del settore, delle autorità governative e dei pazienti è solo l'inizio della soluzione. Rendere la supplementazione nutrizionale parte integrante e strutturale della gestione delle malattie - con l'utilizzo di tecnologie appropriate - è l'altra parte della soluzione del problema.

Nei contesti sanitari dei paesi occidentali, la prevalenza della malnutrizione è allarmante. I pazienti degenti negli Istituti di ricovero mostrano sintomi di malnutrizione fino all' $85 \%$ dei casi, mentre negli ospedali la prevalenza sale a volte al $60 \%$. Percentuali rilevanti sono state osservate anche a livello domiciliare. $\underline{\text { Gli studi }}$ mostrano che fino al $40 \%$ dei pazienti anziani e quasi un quinto di tutti i pazienti di medicina generale soffrono di malnutrizione come conseguenza della propria malattia. Ulteriori ricerche suggeriscono risultati simili in un'ampia gamma di malattie specifiche. Spesso i pazienti sono già in stato di malnutrizione quando vengono ricoverati in ospedale, il che indica che il problema nasce a livello domiciliare.

Gli specialisti in molti casi riconoscono già la malnutrizione ed il calo ponderale come segnali di una perdita dello stato di salute del paziente. Il solo suggerimento al paziente di aumentare l'assunzione di nutrienti assumendo maggiori quantità di cibo si è dimostrato inefficace. Mangiare anche solo un pasto leggero per molti pazienti è uno sforzo sovrumano. I pazienti si trovano intrappolati in un circolo vizioso, per cui la malattia causa perdita dell'appetito, che a sua volta determina una minore assunzione di cibo e di conseguenza un peggioramento della condizione fisica e mentale, che ancora una volta determina la riduzione dell' appetito e così via.

Diventa quindi necessario ricorrere ad una supplementazione nutrizionale in forma di alimenti facilmente somministrabili. I supplementi nutrizionali orali (SNO), così come la nutrizione enterale per sonda (NES) si sono dimostrati efficaci nel mantenere o nel migliorare lo stato nutrizionale del paziente. Purtroppo, sembra che i sistemi sanitari nazionali non incoraggino né consentano l'implementazione del- 
la supplementazione nutrizionale come elemento strutturale nella gestione delle malattie.

Sulla base dei dati raccolti si può concludere che le conseguenze negative segnalate non avvengono né in maniera casuale, né raramente, ma sono gravi problemi strutturali che richiedono un' azione mirata alla loro risoluzione. Gli studi esaminati hanno dimostrato che la malnutrizione associata a patologie determina maggiori percentuali di mortalità e di morbilità, una sopravvivenza più breve, il peggioramento della qualità di vita, la formazione di dolorose piaghe da decubito, una ridotta capacità funzionale, minori risposte terapeutiche, maggior numero di prescrizioni, maggior numero di visite mediche e, in ultimo, ma non meno importante, il prolungamento delle degenze ospedaliere.

È chiaro che tutto ciò diventa gravoso per il paziente e per il personale assistenziale, sottoposto a una maggiore pressione lavorativa che cresce con l'aumento del numero di pazienti malnutriti da accudire. Anche i sistemi sanitari nazionali risentono pesantemente dell'aumento dei costi dovuto alla malnutrizione. Negli USA è stato calcolato che in alcuni casi il costo per paziente può raggiungere la cifra di 14.000 dollari.

Gli effetti positivi che si ottengono con i supplementi nutrizionali per os (SNO) o con la nutrizione enterale (NES) per sonda comprendono l'incremento ponderale e l' aumento della massa muscolare, determinando miglioramenti funzionali quali un migliore tono muscolare, l'aumento della capacità di compiere determinate attività (ad esempio percorrere distanze più lunghe) ed il miglioramento della salute fisica e mentale, con conseguente miglioramento della qualità di vita. Questi risultati sono direttamente correlati al maggiore apporto di energia e di nutrienti ed alla maggiore assunzione volontaria di cibo. Oltre a questi benefici funzionali, i miglioramenti dei risultati clinici dovuti all'intervento nutrizionale determinano una minore percentuale di mortalità, degenze ospedaliere più brevi e la riduzione delle complicanze.

È già stato affermato che l'intervento nutrizionale è vantaggioso non solo per i pazienti ed il personale assistenziale, ma anche per il Sistema Sanitario Nazionale in quanto è stata determinata la sua efficacia in termini di costo-beneficio sui pazienti malnutriti. Alcuni studi hanno prodotto risultati inequivocabili: l'intervento con prodotti nutrizionali specifici può determinare risparmi di diverse migliaia di dollari per paziente. Le ragioni principali di questo risparmio sono dovute a un minore utilizzo delle strutture sanitarie, grazie a degenze ospedaliere più brevi, ad un minore ricorso allo specialista ed alla riduzione dei costi di trattamento delle complicanze, quali le piaghe da decubito. I costi degli SNO e della NES sono insignificanti se confrontati con i risparmi che questi trattamenti possono potenzialmente generare.

Dalle informazioni disponibili risulta chiaramente l'opportunità di considerare l'introduzione dell'intervento nutrizionale (per sonda e per os) come parte strutturale del trattamento, e come tale rimborsabile da parte del SSN.

Un approccio congiunto, in cui agli sforzi del personale medico e assistenziale si affianchi una responsabilizzazione del paziente, istruito al monitoraggio del proprio apporto e stato nutrizionale, può sicuramente contribuire a ridurre l'impatto clinico ed economico della malnutrizione associata a patologie.

\section{BIBLIOGRAFIA}

1. Widhalm K, Lackner B, Bauernfried M. Medical education in nutrition in Europe. Workshop. Ann Nutr Metab. 1997;41(1):66-8.

2. Stratton RJ. Summary of a systematic review on oral nutritional supplement use in the community. Proc Nutr Soc. 59: 469-76, 2000.

3. Elia M, Stratton RJ. How much undernutrition is there in hospitals? Br J Nutr 84: 257-9, 2000.

4. Stratton RJ, Elia M. Are oral nutritional supplements of benefit to patients in the community? Findings from a systematic review. Curr Opin Clin Nutr Metab Care 3: 311-5, 2000.

5. Stratton RJ, Elia M. The effects of enteral tube feeding and parenteral nutrition on appetite sensations and food intake in health and disease. Clin Nutr. 18: 63-70, 1999.

6. WHO. Physical status: the use and interpretation of anthropometry . Geneva: WHO technical report series 854 , WHO, 1995. 
7. Green CJ. Existence, causes and consequences of disease-related malnutrition in the hospital and the community, and clinical and financial benefits of nutritional intervention. Clinical Nutrition 18 (Suppl 2): 3-28, 1999.

8. The European commision. Key data on health 2000. Luxembourg: Eurostat 2001

9. Wilson MM, Vaswani S, Liu D, Morley JE, Miller DK Prevalence and causes of undernutrition in medical outpatients. Am J Med 104:56-63, 1998

10. Gallagher-Allred CR et al. Malnutrition and clinical outcomes: The case for for medical nutrition therapy. Journal of the American Dietetic Association, 96: 361-6, 369, 1997.

11. Gallagher-Allred CR, Voss AC, Koop KL. The effect of medical nutrition therapy on malnutrition and clinical outcomes. Nutrition, 15(6):512-4,1999.

12. Chima CS et al. Relationship of nutritional status to length of stay, hospital costs, and discharge status of patients hospitalized in the medicine service. Journal of the American Dietetic Association, 97: 975-8, 1997.

13. Allison SP Malnutrition, disease, and outcome. Nutrition 16:590-3, 2000

14. Martyn CN, Winter PD, Coles SJ, Edington J. Effect of nutritional status on use of health care resources by patients with chronic disease living in the community. Clin Nutr 17:119-23, 1998.

15. Mowe M, Bohmer T Nutrition problems among home-living elderly people may lead to disease and hospitalization. Nutr Rev 54:S22-4, 1996

16. Hall K, Whiting SJ, Comfort B Low nutrient intake contributes to adverse clinical outcomes in hospitalized elderly patients. Nutr Rev 58:214-7, 2000

17. Kaminski MV Jr Impact of nutrition support on patient outcome and hospital costs. J Can Diet Assoc 49:85-8, 1988

18. Edington J, Kon P Prevalence of malnutrition in the community. Nutrition 13:238-40, 1997.

19. Black DR, Sciacca JP, Coster DC Extremes in body mass index: probability of healthcare expenditures. Prev Med. 23(3):385-93, 1994.

20. Heithoff KA, Cuffel BJ, Kennedy S, Peters J The association between body mass and health care expenditures. Clin Ther. 19(4):811-20, 1997

21. The Nutrition Screening Initiative. The clinical and cost-effectiveness of medical nutrtion therapy: evidence and estimates of potential medicare savings from the use of selected nutrition interventions. Washington DC: Nutrition Screening Initiative, 1996.

22. Krondl M, Coleman PH, Bradley CL, Lau D, Ryan N. Subjectively healthy elderly consuming a liquid nutrition supplement maintained body mass index and improved some nutritional parameters and perceived well-being. $\mathrm{J}$ Am Diet Assoc 99:1542-8, 1999.

23. Schwenk A, Steuck H, Kremer G. Oral supplements as adjunctive treatment to nutritional counseling in malnourished HIV-infected patients: randomized controlled trial. Clin Nutr 18:371-4, 1999.

24. Verma S, Kirkwood B, Brown S, Giaffer MH. Oral nutritional supplementation is effective in the maintenance of remission in Crohn's disease. Dig Liver Dis 32:769-74, 2000.

25. Pennington CR Disease and malnutrition in British hospitals. Proc Nutr Soc 56:393-407, 1997.

26. Pennington CR Malnutrition in hospital practice. Nutrition 12:56-7, 1996.

27. Lehmann AB Review: undernutrition in elderly people. Age Ageing. 18:339-53, 1989

28. Detsky AS, Smalley PS, Chang J Is this patient malnourished? JAMA. 271:54-8, 1994.

29. Pennington CR Malnutrition in hospital: the case of the stroke patient. Br J Nutr 79:477-8, 1998.

30. Pennington CR, McWhirter JP Patients go hungry in British hospitals. Malnutrition is common, unrecognised, and treatable in hospital patients. BMJ 314:752,1997.

31. Joosten E, Vanderelst B, Pelemans W The effect of different diagnostic criteria on the prevalence of malnutrition in a hospitalized geriatric population. Aging 11:390-4, 1999.

32. Hartley GH Nutritional support in renal failure. Proc Nutr Soc 59:485-6, 2000.

33. Buchholz DW, Neumann S A randomized, controlled, single-blind trial of nutritional supplementation after acute stroke. Dysphagia 14:185, 1999

34. Malone M Quality of life of patients receiving home parenteral or enteral nutrition support. Pharmacoeconomics 5:101-8, 1994. 
35. Johnson LE, Dooley PA, Gleick JB Oral nutritional supplement use in elderly nursing home patients. J Am Geriatr Soc 41:947-52, 1993

36. Elia M Artificial nutritional support in clinical practice in Britain. J R Coll Physicians Lond 27:8-15, 1993.

37. Cole L Early enteral feeding after surgery. Crit Care Nurs Clin North Am 11:227-31, 1999

38. Hedberg AM, Lairson DR, Aday LA, Chow J, Suki R, Houston S, Wolf JA Economic implications of an early postoperative enteral feeding protocol. J Am Diet Assoc 99:802-7, 1999.

39. Goff KL Cost and cost-benefit of enteral nutrition. Gastrointest Endosc Clin N Am 8:733-44, 1998

40. Tchekmedyian NS Pharmacoeconomics of nutritional support in cancer. Semin Oncol.;25 (Suppl 6):62-9, 1998

41. Reilly JJ Jr, Hull SF, Albert N, Waller A, Bringardener S Economic impact of malnutrition: a model system for hospitalized patients. J Parenter Enteral Nutr 12:371-6, 1988.

42. Gregory J, Foster K, Tyler H, Wiseman M. The dietary and nutritional survey of British adults. London: Office of Population Censuses and Surveys. Her Majesty's Stationary Office, 1990.

43. OPCS. Health survey for England 1992. London: HMSO, 1994.

44. Finch S et al. National diet and Nutrition Survey: people aged 65 years and over, 1: Report of the Diet and Nutrition Survey. London: Office of Population Censuses and Surveys. Her Majesty's Stationary Office, 1998.

45. Thorslund S et al. Prevalence of protein-energy malnutrition in a large population of elderly people at home. Scandinavian Journal of Primary Health Care 8: 243-8, 1990.

46. Harvard School of Public Health on behalf of the WHO and the World Bank. Global Health Statistics: A compendium of incidence, prevalence and mortality estimates for over 200 conditions. Boston: Harvard University Press, 1996 